\title{
EXAMINATION OF THE EFFICACY OF PROXIMITY WARNING DEVICES FOR YOUNG AND OLDER DRIVERS
}

\author{
Arthur F. Kramer, Nicholas D. Cassavaugh, \\ William J. Horrey, Ensar Becic, Jeffery Mayhugh \\ Beckman Institute and Department of Psychology \\ University of Illinois \\ 405 N. Mathews Avenue, Urbana, Illinois, USA \\ E-mail: akramer@s.psych.uiuc.edu
}

PAPER NOT AVAILABLE

\begin{abstract}
\section{OBJECTIVES}

The study was conducted to examine the efficacy of uni- and multi-modal proximity warning devices for forward object collision and side-object detection for young and older adult drivers.
\end{abstract}

\section{METHODS}

Two experiments were conducted, each with 20 young (18 to 30 years of age) and 20 older (61 to 80 years of age) healthy and high functioning drivers. In each, participants drove a series of brief ( $\sim 4$ minute) highway scenarios with temporally unpredictable forward and side collision events (i.e., other vehicles). The experiments were conducted in a fixed-base Drive Safety simulator with a 135-degree wrap-around forward field and a 135-degree rear field. Light crosswinds were included in Experiment 1, while heavier crosswinds were introduced in the second experiment. A secondary visual read-out task from an in-vehicle LCD display was also included in the second experiment.

In Experiment 1, potential collision events were signaled 2.2 seconds before impact by visual, auditory, auditory+visual or tactile+visual warnings that were spatially mapped to the location of the obstacle (left, right or center). A control condition in which subjects drove without any proximity warning device was also included in the experiment. Experiment 2 included the control, auditory+visual and visual warnings from Experiment 1.

A number of dependent measures were collected, including velocity, lane position, steering wheel movement, brake and accelerator position. However, we will focus on the response time (as measured by steering wheel deflections or removal of the foot from the accelerator) to potential collision events as well as the number of collisions in different experimental conditions.

\section{RESULTS}

In both Experiments 1 and 2, the auditory+visual warning device produced the most rapid response and also resulted in the fewest collisions. The reduction in response time and collisions, relative to the no-warning control condition was larger in Experiment 2 than in Experiment 1, 
likely as a result to the more challenging driving scenarios (with the higher and unpredictable winds and introduction of the secondary task) in this experiment.

Older adults responded just as quickly as younger adults to the potential collision events in both of the experiments. This is a very surprising finding given a voluminous laboratory literature, which suggests that older adults display slower responses than younger adults on almost any task that has been examined in the laboratory.

In an effort to understand the age-equivalent response times to collision events, we asked young and older participants from the first experiment to take part in an additional experimental session in which they made simple and choice responses to visual and auditory events in a sound attenuated subject booth. Older adults were substantially $(\sim 35 \%)$ slower in each of these simple and choice tasks performed in the laboratory.

Older adults displayed the same performance benefits (in terms of speeded response time and reductions in collisions) from the proximity warning devices, and particularly the auditory+visual device, in both of the experiments as younger adults. However, in Experiment 2, older adults displayed these benefits by neglecting the number read-out secondary task.

\section{CONCLUSIONS}

There are several important conclusions from the present study. First, proximity warning devices, and particularly auditory+visual devices, can substantially speed response time and reduce potential collisions in simulated driving. This is an important observation that has the potential to reduce automobile accidents. Second, both younger and older adults benefit from the proximity warning devices. Such a finding suggests, that at least for individuals with normal vision and hearing, these devices might have substantial utility across a wide variety of drivers. Third, quite to our surprise, older adult drivers responded just as quickly, with and without the proximity warning devices, to potential collision events as younger drivers. Interestingly, ageequivalence in response time to potential collisions was not observed in simple and choice auditory and visual laboratory response time tasks. Such data tentatively suggests that experience and expertise in driving may act as a moderator of age-related decline in general slowing. Given the unpredictable nature of the potential collision events in our study, older drivers may be capitalizing on high levels of vigilance and attentional focus on driving relevant tasks to maintain their ability to rapidly respond to collision events. This hypothesis is supported, in part, by the decrements in secondary task performed observed for the older but not for the younger adults in Experiment 2.

The results from the present study are encouraging both with respect to the utility of proximity warning devices as a means to enhance driver safety as well as for their potential application to drivers of different ages and experience levels. However, clearly additional research will be needed to verify these results in more challenging simulator and on the road driving situations. 